\title{
ASPECTOS TÉCNICOS DA RESSONÂNCIA MAGNÉTICA DE MAMA COM MEIO DE CONTRASTE: REVISÃO DA LITERATURA*
}

\author{
Denise de Deus Leopoldino ${ }^{1}$, Giuseppe D'lppolito ${ }^{2}$, Alexandre Sérgio de Araújo Bezerra ${ }^{3}$, \\ Tatiana Schiller Gracio ${ }^{4}$
}

\begin{abstract}
Resumo Com a difusão do uso de meios de contraste, avanços na tecnologia das bobinas de superfície e desenvolvimento de protocolos rápidos de aquisição de imagens, a ressonância magnética (RM) de mama com meio de contraste tem-se mostrado importante modalidade na detecção, diagnóstico e estadiamento do câncer de mama. Apesar desses avanços, existem alguns pontos não consensuais no que diz respeito aos aspectos técnicos e critérios de interpretação de imagem da RM contrastada de mama. Neste artigo fazemos revisão bibliográfica dos parâmetros de interpretação de imagens e aspectos técnicos da RM de mama, incluindo considerações sobre a "performance" do equipamento, bobinas de radiofreqüência dedicadas, modo de utilização de contraste paramagnético, técnicas de supressão de gordura, planos de aquisição, seqüências de pulso e fontes de artefato.

Unitermos: Ressonância magnética; Mamas; Técnicas de imagem; Câncer de mama.
\end{abstract}

Abstract Technical aspects of contrast-enhanced magnetic resonance imaging of the breast: literature review. With the advances in surface coil technology and the development of new imaging protocols in addition to the increase of the use of contrast agents, contrast enhanced magnetic resonance imaging (MRI) has emerged as a promising modality for detection, diagnosis and staging of breast cancer. Despite these advances, there are some unresolved issues, including no defined standard technique for contrast-enhanced breast MRI and no standard criteria of interpretation for the evaluation of such studies. In this article, we review the literature and discuss the general requirements and recommendations for contrast agent-enhanced breast MRI, including image interpretation criteria, MR equipment, dedicated radiofrequency coils, use of paramagnetic contrast agents, fat-suppression techniques, planes of acquisition, pulse sequence specifications and artifact sources.

Key words: Magnetic resonance imaging; Breasts; Imaging techniques; Breast neoplasms.

\section{INTRODUÇÃO}

Nas duas últimas décadas, vários avanços foram obtidos no campo da ressonância magnética (RM) de mama, como o uso sistemático do meio de contraste e avanço na tecnologia das bobinas de superfície, tornando-a importante modalidade de imagem na detecção, diagnóstico e estadiamento do câncer de mama ${ }^{(1-4)}$.

Mesmo com todos esses avanços, a RM das mamas para diagnóstico e acompanha-

* Trabalho realizado na Scopo Diagnóstico, Serviço de US/TC RM do Hospital São Luiz, São Paulo, SP.

1. Médica do Setor de Tomografia e Ressonância Magnética do Hospital São Luiz.

2. Médico do Setor de Diagnóstico por Imagem do Hospital São Luiz, Docente do Departamento de Diagnóstico por Imagem da Universidade Federal de São Paulo/Escola Paulista de Medicina (Unifesp/EPM).

3. Médico do Setor de Diagnóstico por Imagem do Hospital São Luiz, Pós-graduando do Departamento de Diagnóstico por Imagem da Unifesp/EPM.

4. Biomédica do Setor de Tomografia e Ressonância Magnética do Hospital São Luiz.

Endereço para correspondência: Dra. Denise de Deus Leopoldino. SQN 313, Bloco H, ap. 306. Brasília, DF, 70766-080. E-mail: leopoldinod@yahoo.com

Recebido para publicação em 3/3/2004. Aceito, após revisão, em 22/6/2004. mento do câncer de mama ainda é um método em desenvolvimento. Existem alguns pontos que até o presente momento permanecem controversos na literatura, como, por exemplo, protocolos de exame bem definidos, critérios de interpretação de imagens e indicações clínicas para o uso da $\mathrm{RM}^{(1,3-6)}$.

Neste artigo fazemos revisão bibliográfica da literatura científica publicada sobre RM de mama, com foco nos aspectos técnicos de aquisição de imagem e parâmetros de interpretação, e ilustramos com casos selecionados entre mais de 200 exames de RM de mama realizados no nosso serviço e indicados principalmente para detecção e caracterização de nódulos mamários.

\section{CARACTERÍSTICA DO EQUIPAMENTO}

A maioria dos estudos de imagem por RM das mamas relatados na literatura tem sido realizada com equipamentos de alto campo $(1,0-1,5 \mathrm{~T})^{(\mathbf{1}, \mathbf{4 , 5})}$. Isto se deve à ele- vada relação sinal-ruído, ao melhor desempenho na técnica com supressão de gordura e maior definição do realce pelo meio de contraste nos equipamentos de alto campo ${ }^{(1,5,7)}$.

Equipamentos de baixo campo possuem inerentemente menor relação sinal-ruído e, portanto, há maior comprometimento da resolução espacial e temporal. Outra limitação em relação aos aparelhos de baixo campo está relacionada às técnicas de supressão de gordura. Nestes equipamentos a capacidade de separação dos picos de gordura e água encontra-se diminuída, tornando a "spectrally selected fat suppression" de difícil realização ${ }^{(\mathbf{1})}$. Outro aspecto, ainda mais importante, diz respeito à diferença entre as características do realce. Os tempos de relaxamento $\mathrm{T} 1$ dos tecidos tendem a cair com a diminuição da intensidade de campo magnético, e desta maneira há menor modificação (encurtamento) do T1 pelo gadolínio. O resultado disto é que a diferença de contraste entre o tecido normal e o contrastado será menor para 
uma determinada concentração de gadolínio em seqüências ponderadas em T1 obtidas em equipamentos operando em baixo campo $^{(\mathbf{1})}$.

Kuhl et al., ao analisarem 42 pacientes portadoras de lesões nodulares mamárias submetidas à RM de mama em magneto de 1,5T utilizando seqüência gradiente-eco 2D e seqüência gradiente-eco 3D em magneto de $0,5 \mathrm{~T}$, demonstraram que a qualidade das imagens era comparável em ambos os equipamentos ${ }^{(8)}$. Não encontramos, na literatura, outros trabalhos que sustentem esses resultados.

Equipamentos de alto campo apresentam "performance" semelhante no diagnóstico de lesões mamárias, como foi demonstrado por Heywang-Köbrunner et al. através de estudo multicêntrico, que obtiveram resultados superponíveis quando utilizaram equipamentos de 1,0 e 1,5T ${ }^{(\mathbf{9})}$.

\section{BOBINAS}

A utilização de bobinas dedicadas determina aumento importante da relação sinal-ruído, cujos resultados podem ser considerados um fator crítico na qualidade das imagens por RM. Uma melhor relação sinal-ruído permite obter cortes mais finos, menor campo de visão e melhor resolução de imagem ${ }^{(\mathbf{1 0})}$. Estes são fatores cruciais na obtenção de imagens da mama e por esses motivos bobinas específicas devem ser utilizadas sempre que possível na obtenção de imagens de RM das mamas ${ }^{(2-5,7)}$.

Diversos tipos de bobinas para estudo específico das mamas têm sido utilizados, como as bobinas de superfície e as bobinas de sinergia ("phased-array multicoils"). Em relação ao “design”, estas podem ser unilaterais ou bilaterais ${ }^{(\mathbf{1})}$. Na experiência pessoal de Orel e Schnall a "performance" da bobinas de sinergia bilateral é superior à das bobinas de superfície ${ }^{(\mathbf{1})}$ (Figura 1).

Existem, ainda, algumas bobinas que permitem acesso à realização de intervenções guiadas por RM, o que pode ser particularmente útil para acessar lesões que foram identificadas apenas em exames de RM e não visualizadas em mamografias ou estudos ultra-sonográficos (Figura 2). Nestes casos, é importante observar que, além da bobina específica, é necessária agulha não ferromagnética ${ }^{(\mathbf{1}, 4,11-13)}$.

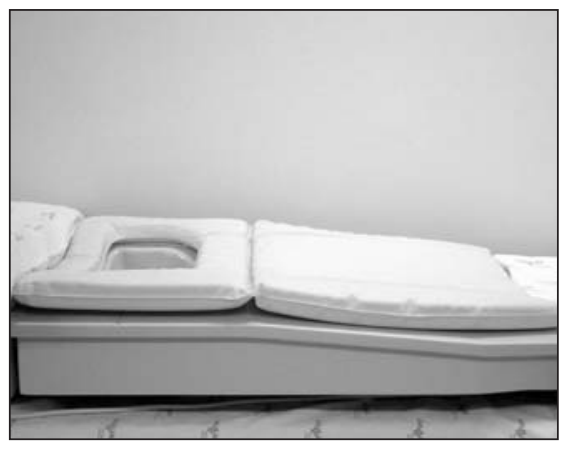

A

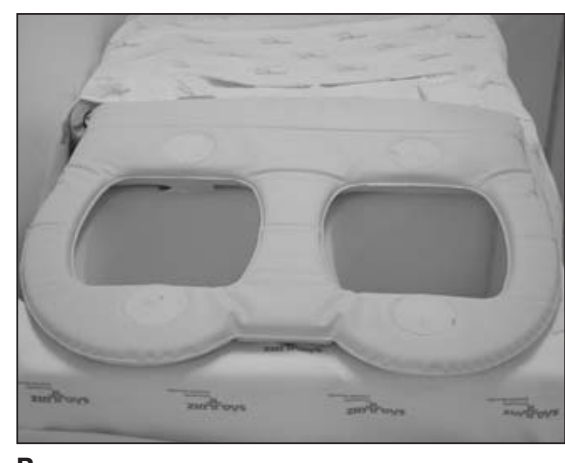

Figura 1. A: Bobina de superfície. B: Bobina de sinergia, com possibilidade de encaixe de ambas as mamas. Paciente posicionada em posição prona.

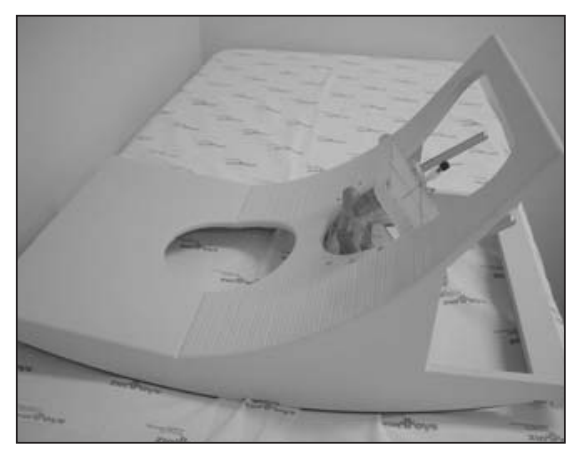

Figura 2. Suporte específico para intervenção dirigida através de RM. Nota-se o compressor e a grade quadriculada que permite o acesso da agulha.

São poucos os estudos que avaliam o uso de diversos tipos de bobina de mama. Em recente estudo comparativo entre cinco tipos de bobinas de mama, foram analisados os seguintes fatores: relação sinalruído, uniformidade de campo, conforto, posicionamento do paciente, acesso às mamas e opção de imagem unilateral. A bobina de sinergia com quatro elementos mostrou superior relação sinal-ruído, flexibilidade de imagem e acesso a procedimentos intervencionistas. Não houve consenso quanto à uniformidade de campo e conforto da paciente nas bobinas utilizadas $^{(\mathbf{1 3 )}}$. Assim, parece apropriado recomendar o uso de bobinas de sinergia, para exames de RM de mama, quando disponíveis.

\section{USO DE CONTRASTE PARAMAGNÉTICO}

O uso do contraste paramagnético no estudo por RM das mamas mostrou-se importante na detecção e caracterização do câncer de mama. O uso intravenoso do contraste que passa do espaço extravascular e se acumula em tecidos com rica vascularização aumentou a sensibilidade e especificidade da investigação ${ }^{(3)}$. A justificativa para seu uso é que a maioria dos cânceres apresenta realce consistente após a injeção intravenosa do contraste paramagnético ${ }^{(3,5)}$. O substrato fisiopatológico para sustentar esta afirmação está relacionado ao fato de que carcinomas com dimensões maiores que $3 \mathrm{~mm}$ secretam fatores angiogênicos, com formação de neovascularização e anastomoses arteriovenosas. Este processo resulta em um acúmulo mais rápido de contraste em cânceres do que lesões benignas ${ }^{(3,5-7,14)}$.

Os meios de contraste mais comumente utilizados em RM têm como base o gadolínio $^{(\mathbf{1 5})}$. A versão mais utilizada entre os artigos analisados é o gadopentetato dimeglumina (Gd-DTPA) ${ }^{(\mathbf{6 , 7 , 9 , 1 6 - 2 3 )}}$. O meio de contraste é administrado por via intravenosa, freqüentemente em "bolus" e seguido ou não por injeção de solução salina. A dose administrada varia entre $0,1 \mathrm{mmol} / \mathrm{kg}$ e $0,2 \mathrm{mmol} / \mathrm{kg}$ nos diversos estudos anali$\operatorname{sados}^{(3,5,24,25)}$.

Alguns autores, como Heywang-Köbrunner et al., demonstraram maior conspicuidade de lesões malignas com doses de $0,16 \mathrm{mmol} / \mathrm{kg}$ quando comparadas com doses de $0,1 \mathrm{mmol} / \mathrm{kg}$ de gadolínio $^{(21)}$, ou seja, utilizando uma vez e meia a dose usualmente preconizada. Por outro lado, esses resultados não foram replicados por outros autores.

Mais recentemente, Knopp et al. demonstraram que o gadobenato dimeglumina (Gd-BOPTA) pode ser utilizado como meio de contraste efetivo na detecção e 
caracterização do câncer de mama por RM. Através de estudo comparativo em relação à dose administrada, os resultados desses autores sugerem que a dose de 0,1 $\mathrm{mmol} / \mathrm{kg}$ parece oferecer vantagens em relação às doses de 0,05 e $0,2 \mathrm{mmol} / \mathrm{kg}$ de Gd-BOPTA. Foram também comparados os resultados em relação à dose de 0,1 mmol/kg de Gd-BOPTA e Gd-DTPA e os resultados com o Gd-BOPTA foram superiores ${ }^{(\mathbf{2 6})}$. Porém, acreditamos que outros estudos mostram-se necessários para validar esses resultados.

\section{PLANO DE AQUISIÇÃO}

Não existe consenso em relação ao melhor plano de aquisição para o estudo por RM de mama. Ao realizar o estudo de uma única mama qualquer plano de aquisição pode ser utilizado, embora o plano sagital seja o mais freqüentemente empregado. $\mathrm{O}$ exame bilateral é mais freqüentemente realizado nos planos axial ou coronal ${ }^{(\mathbf{1})}$.

Ao analisar 15 trabalhos publicados entre 1998 e 2003, notamos que $13,3 \%(2 / 15)$ dos autores utilizam somente o plano axial, $13,3 \%$ (2/15) utilizam somente o plano coronal, 20\%(3/15) utilizam mais de um plano de aquisição e 53,3\% (8/15) utilizam apenas o plano sagital ${ }^{(6,7,9,16,18-20,22,23,26-31)}$

Alguns autores acreditam que o plano sagital permite melhor definição que o $\operatorname{axial}^{(1,7,16,22,23,30,31)}$, inclusive em função de menor quantidade de artefatos de batimentos cardíacos que podem se projetar sobre a mama. A limitação deste plano está relacionada ao fato de apenas poucos equipamentos atualmente permitirem a aquisição simultânea e dinâmica das imagens no plano sagital em ambas as mamas, o que reduz a utilidade do exame no rastreamento de neoplasia bilateral quando se utiliza a fase contrastada.

\section{SUPRESSÃO DE GORDURA}

A gordura exibe alta intensidade de sinal nas imagens ponderadas em T1 e pode interferir na identificação de pequenas áreas de realce (Figura 3). Dessa forma, a supressão do sinal da gordura é importante na identificação de lesões nodulares focais. A supressão do sinal da gordura pode ser realizada por meio de técnicas de su-
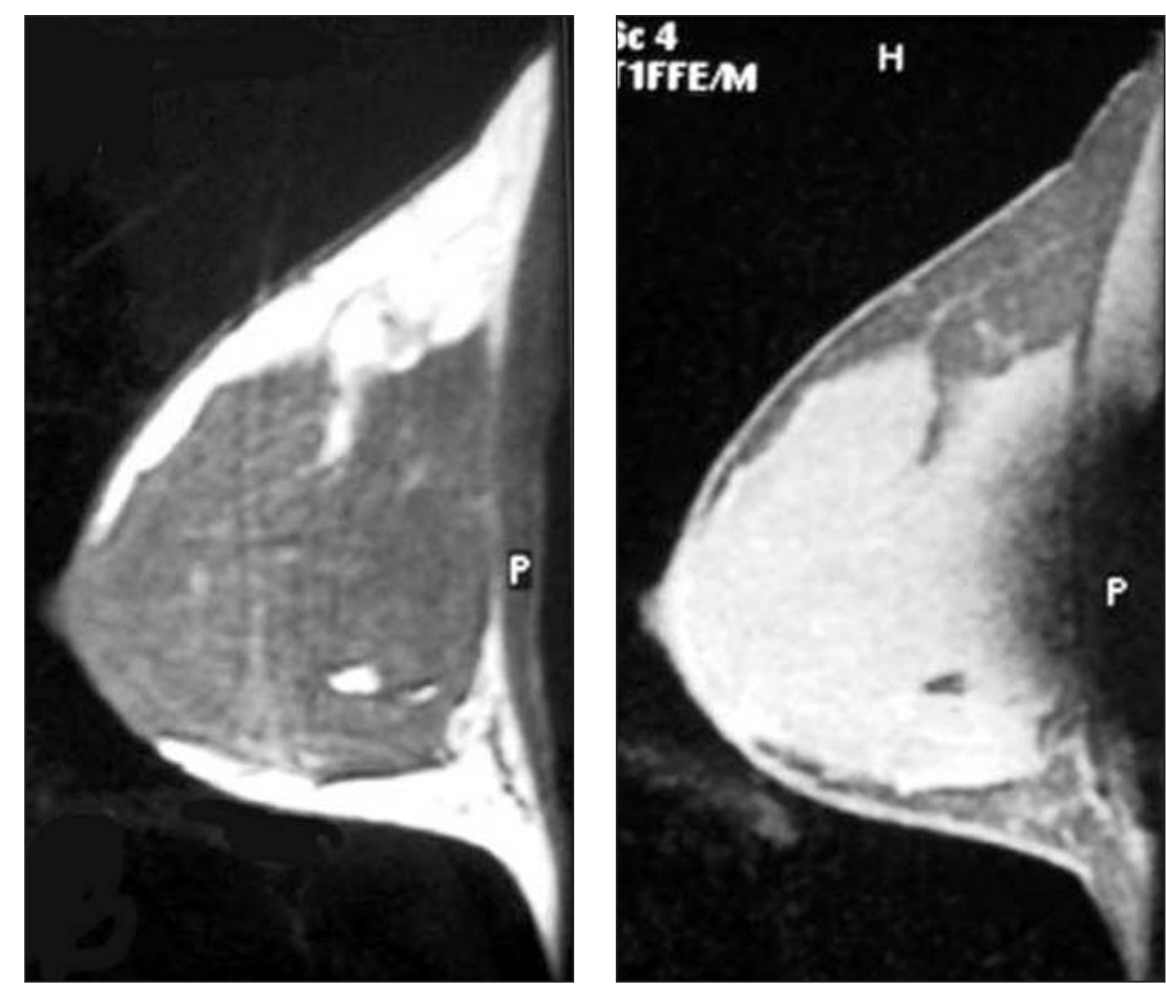

A

B

Figura 3. A: RM no plano sagital ponderada em T1 sem supressão de gordura. Nota-se hipersinal do tecido celular subcutâneo. B: Imagem no plano sagital ponderada em T1 com supressão de gordura. Nota-se redução difusa da intensidade de sinal emitida pelo tecido celular subcutâneo e maior definição do parênquima mamário. É possível observar artefato de inomogeneidade de campo magnético (P).

pressão ou subtração das imagens pré e

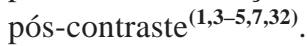

A subtração de imagens possui a desvantagem de ser bastante sensível à movimentação, podendo, dessa forma, resultar em registros errôneos. Por esta razão, a supressão de gordura "spectrally selected fat suppression" é preferencialmente utilizada, com pequeno aumento no tempo de aquisição. A maior vantagem deste método é a baixa interferência da movimentação da paciente na qualidade da imagem. Porém, há uma desvantagem, que é a maior sensibilidade à não homogeneidade do campo magnético (Figura 4), o que pode dificultar, em alguns casos, uma supressão de gordura homogênea e satisfatória ${ }^{(3-5,7,32)}$.

\section{SEQÜÊNCIAS DE PULSO}

Os protocolos de aquisição de RM de mama apresentam bastante variação. O elemento comum das RM de mamas com contraste é a aquisição de imagens ponderadas em T1 antes e após a administração do contraste. As seqüências gradiente-eco substituíram as seqüências spin-eco nos protocolos de aquisição de imagem das mamas, pois são mais rápidas que as seqüências spin-eco, possibilitando a obtenção de imagens com tempos de aquisição mais curtos e maior resolução temporal, permitindo a realização de estudos dinâmicos com curvas de realce ${ }^{(\mathbf{1})}$.

As seqüências gradiente-eco apresentam melhor contraste T1 e são mais sensíveis aos efeitos de encurtamento T1 do gadolínio. Apesar da utilização de ambas as sequiências, tanto as bidimensionais (2D) como as tridimensionais (3D), para a detecção confiável de pequenas lesões ( $<5 \mathrm{~mm}$ ), é consenso que a técnica 3D de alta resolução deve ser usada para se obter uma maior área de cobertura com cortes finos $(\leq 3 \mathrm{~mm})$ e sem intervalos entre os cortes ${ }^{(24)}$.

Existem investigadores que utilizam protocolos de aquisição de imagem com ênfase na resolução temporal. Tais técnicas dinâmicas são designadas para o estudo da 

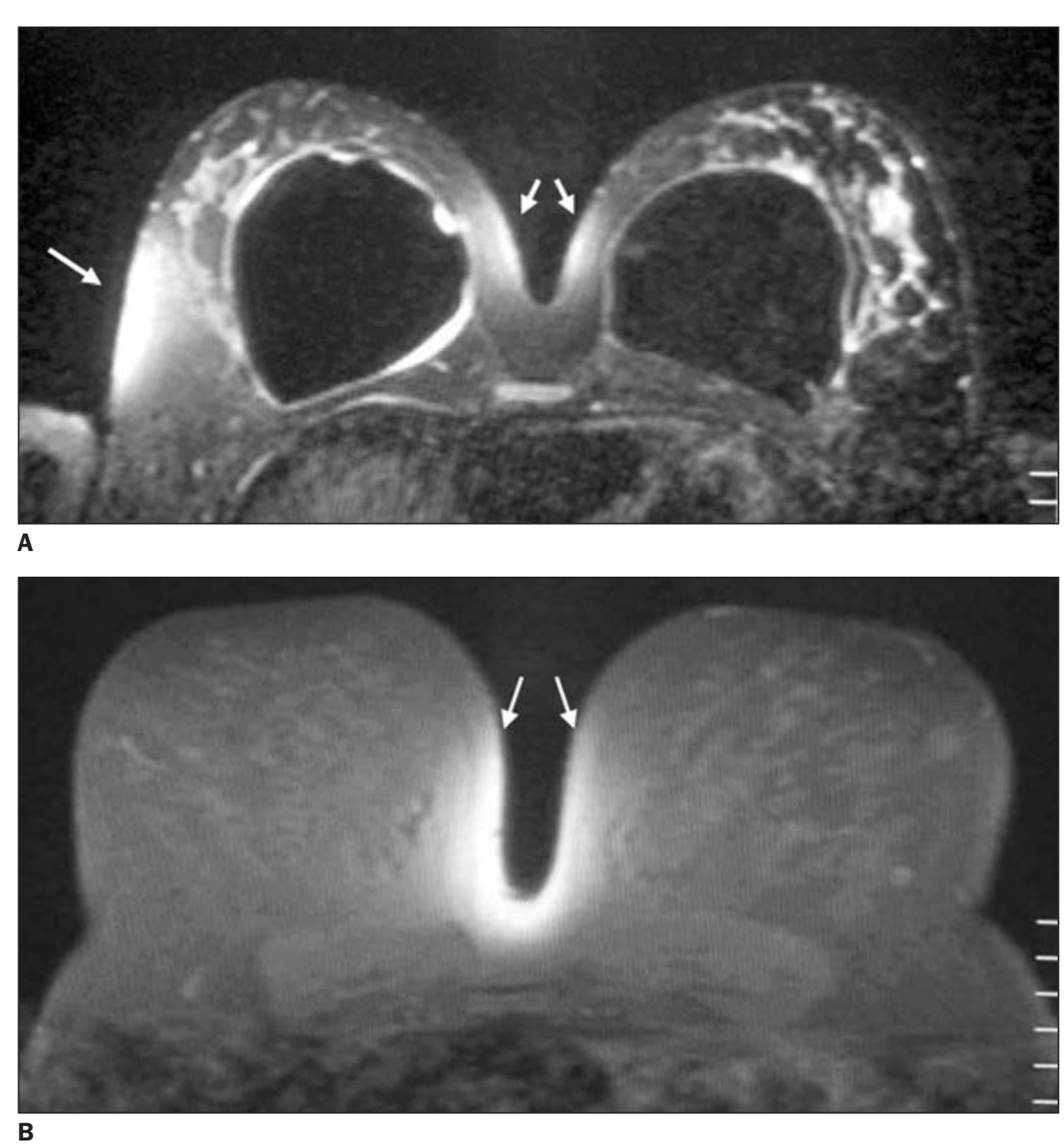

Figura 4. Imagens obtidas no plano axial com supressão de gordura. Nota-se artefato de inomogeneidade de campo caracterizado por hipersinal na periferia nos quadrantes laterais e mediais das mamas (setas).

cinética do realce após a administração do contraste. Essas técnicas de aquisição dinâmicas variam desde de tempos de aquisição de alguns segundos até um minuto. Outros investigadores, no entanto, dão mais ênfase à alta resolução espacial para detectar pequenas lesões e caracterizar sua arquitetura $^{(\mathbf{1})}$.

Avanços tecnológicos permitiram combinar elevada resolução temporal e espacial, inicialmente consideradas excludentes. Atualmente, é possível adquirir imagens com alta resolução espacial e com resolução temporal de aproximadamente 1-2 minutos. Desse modo, obtêm-se imagens com resolução espacial razoável das mamas e que podem ser adquiridas em um período de tempo que permite a realização de pelo menos uma análise qualitativa da cinética do realce ${ }^{(\mathbf{1})}$.

Apesar da RM de mama se fundamentar nas imagens pré e pós-contraste, as tos morfológicos das lesões e características da cinética do realce após a administração do contraste $\mathbf{1}^{(\mathbf{1 , 4 , 6 , 7 , 2 2 , 2 7 )}}$

Não existe padronização do léxico utilizado nas descrições das características das lesões mamárias em relação às características arquiteturais e/ou cinéticas. Vários termos têm sido utilizados na descrição de achados na tentativa de distinguir entre aspectos benignos e malignos ${ }^{(34)}$.

Existem estudos realizados cuja meta é padronizar os termos utilizados na descrição dos achados de RM contrastada das mamas, à semelhança do padrão utilizado nos achados de mamografia, desenvolvido pelo Colégio Americano de Radiologia $\left(\mathrm{BI}-\mathrm{RADS}^{\mathrm{TM}}\right)^{(\mathbf{3 4})}$; no entanto, esta padronização ainda não foi popularizada e difundida. Por outro lado, um esforço tem sido feito neste sentido.

Mediante análise das características morfológicas das lesões focais mamárias, desenvolveu-se um modelo de interpretação dessas lesões ${ }^{(\mathbf{2 3 , 3 5 )}}$. Esses estudos demonstraram que lesões nodulares com contornos lisos (Figura 5) ou lobulados possuem alto valor preditivo negativo para carcinoma (95\% e 90\%, respectivamente). Ao contrário, lesões nodulares que apresentam contornos espiculados e irregulares (Figura 6) possuem alto valor preditivo positivo para malignidade $(91 \%$ e $81 \%$, respectivamente). Em relação ao padrão de realce das lesões mamárias focais, foi demonstrado que a ausência de realce ou presença de mínimo realce apresenta elevado valor preditivo negativo para malignidade (92\%), enquanto o realce anelar possui elevado valor preditivo positivo para malignidade $(86 \%)^{(\mathbf{2 3})}$. Existem também áreas de realce não relacionadas a lesões focais mamárias, como, por exemplo, o realce ductal, que apresenta valor preditivo positivo para malignidade de $85 \%{ }^{(\mathbf{2 3})}$

Fibroadenomas tendem a crescer em vários lóbulos adjacentes, respeitando a demarcação desses lóbulos e desenvolvendo, dessa maneira, septos fibrosos entre os vários lóbulos ${ }^{(4)}$. Portanto, o achado de septações em uma lesão com bordas lisas ou lobuladas pode ser uma boa evidência de benignidade, com valor preditivo negativo para malignidade de $98 \%{ }^{(\mathbf{4 , 2 3})}$.

Porém, características morfológicas de benignidade e malignidade podem coexis- 


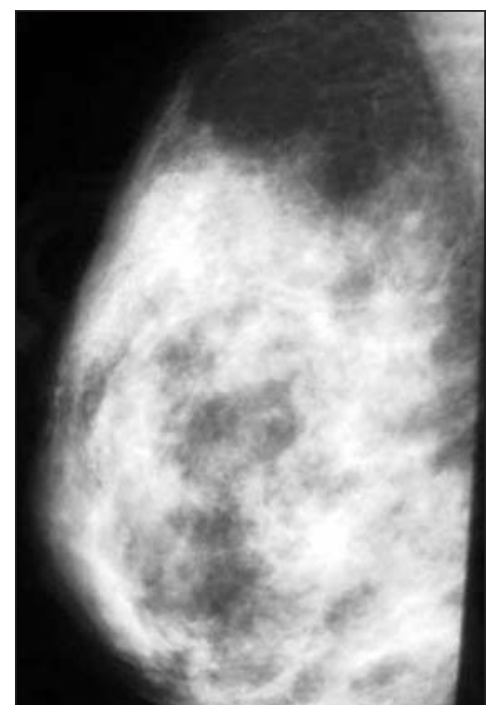
A

Figura 5. A: Mamografia na incidência médio-lateral permite caracterizar mama densa sem evidência de nódulos. B: Ultra-sonografia da mama direita evidencia nódulo hipoecogênico com reforço acústico posterior, não tendo sido possível diferenciar essa lesão entre nódulo sólido com baixa densidade e cisto com líquido espesso. C: RM obtida no plano sagital com imagem ponderada em T2 com supressão de gordura identifica imagem nodular circunscrita homogênea e bem definida, que após injeção endovenosa de contraste paramagnético mostrou intenso realce caracterizando lesão sólida (ver Figura 7).

Figura 6. A: Mamografia na incidência médio-lateral demonstra nódulo parcialmente circunscrito e bem definido, considerado BI-RADS 0. B: RM no plano sagital ponderada em T1 demonstra presença de nódulo homogêneo, porém com contornos relativamente espiculados. Após a injeção endovenosa do contraste paramagnético este nódulo apresentou intenso realce precoce e fugaz (ver Figura 8).

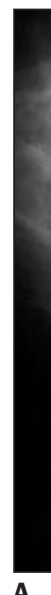

A

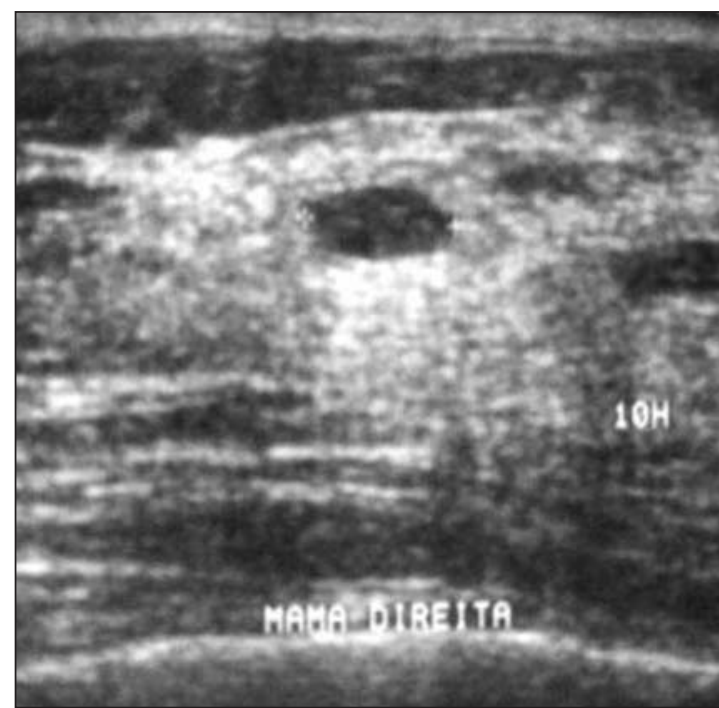

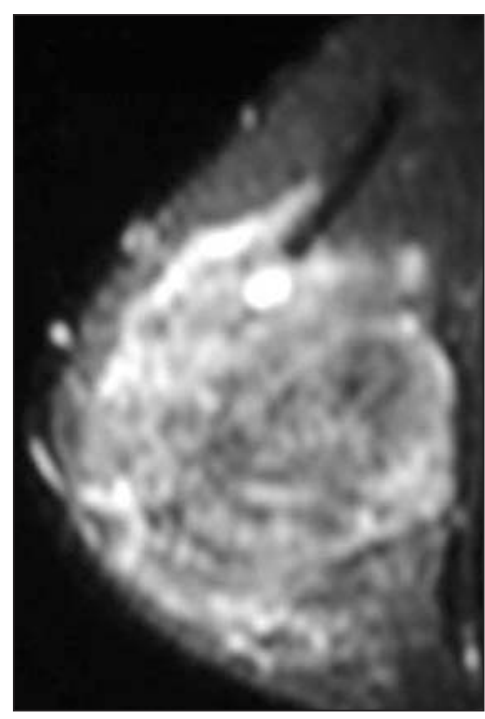

C

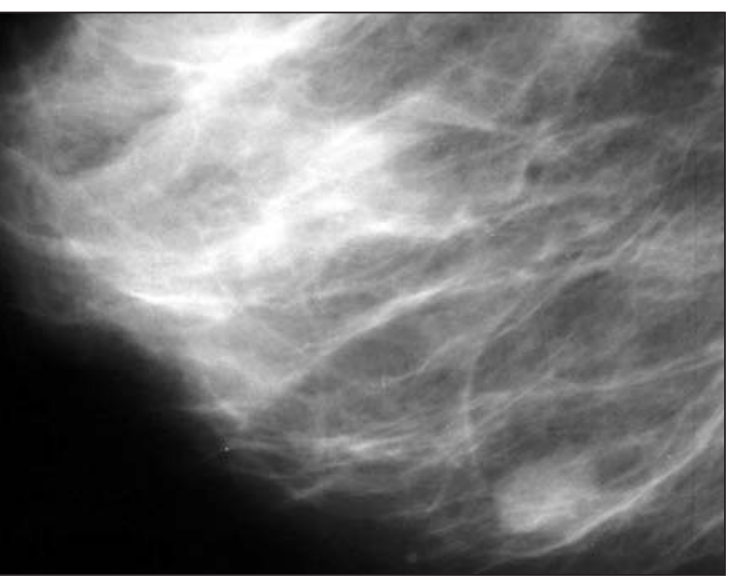

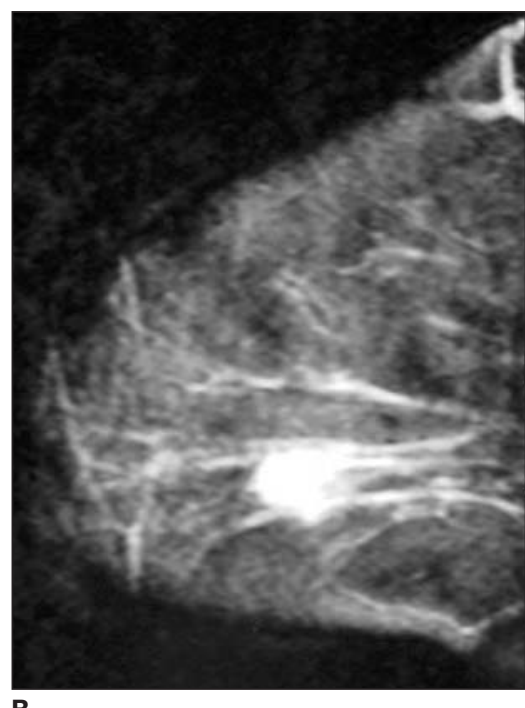

B tir em determinadas lesões nodulares mamárias, e, nestes casos, a característica mais suspeita deve preponderar em relação às demais ${ }^{(7)}$. Este é um conceito bastante subjetivo que pode induzir a erros, e que somente poderão ser reduzidos combinandose a história clínica com os diversos métodos diagnósticos, controles evolutivos precoces e confirmação histológica (sempre que necessário).

Em adição às informações morfológicas, existem características relacionadas à análise cinética do comportamento do con- traste paramagnético em uma lesão mamária. Esta análise pode ser realizada de acordo com parâmetros quantitativos e quali$\operatorname{tativos}^{(\mathbf{1})}$.

Os parâmetros quantitativos têm como objetivo calcular numericamente o aumento da intensidade de sinal após a administração intravenosa do meio de contraste em uma determinada lesão mamária, na tentativa de diferenciar entre lesões benignas e malignas $^{(4)}$, considerando que as lesões malignas apresentam realce mais acentuado e precoce que as lesões benignas ${ }^{(\mathbf{1})}$. Para a realização deste cálculo torna-se necessária a utilização de modelos matemáticos complexos $^{(\mathbf{1})}$. Porém, a relação complexa entre concentração de gadolínio, seqüência de pulso e intensidade de sinal torna extremamente difícil o desenvolvimento de técnicas quantitativas que possam ser generalizadas para equipamentos e técnicas diferentes ${ }^{(4)}$.

Em contraste aos parâmetros quantitativos, foi desenvolvido um método qualitativo pelo qual se pode obter informações sobre as propriedades vasculares de uma 
lesão mamária por meio da análise do aspecto da curva de realce ${ }^{(\mathbf{2 0})}$. O aumento da intensidade de sinal após a administração do contraste é medida em relação ao nível pré-contraste e transformada em gráfico, obtendo-se, dessa maneira, uma curva tempo-intensidade de sinal. Isto requer múltiplos momentos de aquisição, com o primeiro momento no tempo zero quando não há ainda contraste na lesão. Para gerar estes momentos, a mama deve ser examinada continuamente em diversos tempos, após a injeção em "bolus" do contraste ${ }^{(7)}$.

Existem três tipos de padrão de curva tempo-intensidade ${ }^{(\mathbf{2 0})}$. A curva tipo I apresenta realce progressivo e contínuo no decorrer do tempo, indicando que o contraste se acumula na lesão, e é tipicamente observada em lesões benignas (Figura 7). A curva tipo III apresenta rápido clareamento do realce ("washout"), com um pico de realce seguido de declínio, indicando que após a lesão captar o contraste este é rapidamente eliminado, presumivel-

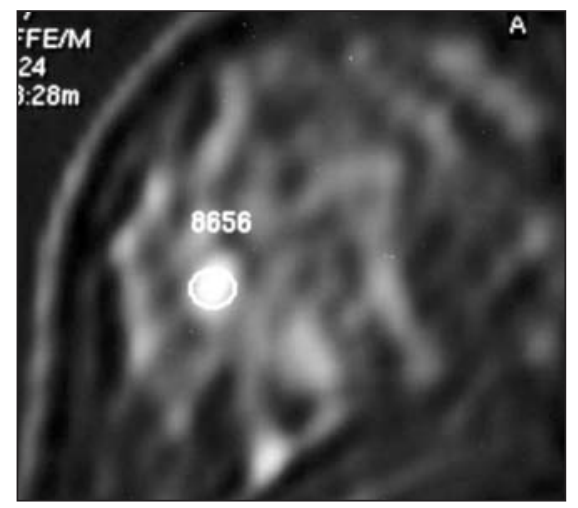
A

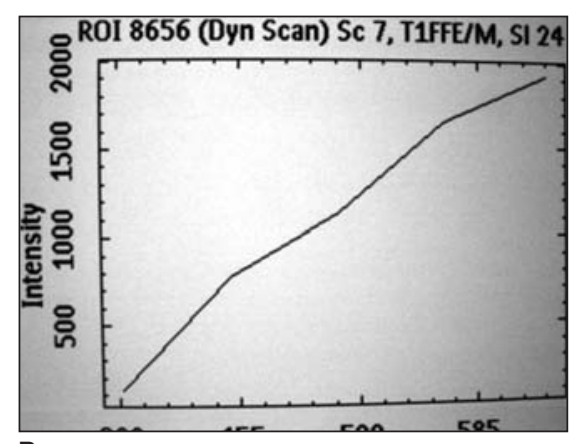

B

Figura 7. A: RM no plano axial ponderada em T1 obtida oito minutos após injeção endovenosa do contraste paramagnético, com medida de intensidade de sinal. B: A curva de realce obtida a partir da análise de oito seqüências consecutivas demonstra realce progressivo e persistente no decorrer do tempo indicando curva tipo I. mente pela presença de vasos colaterais, capilares e anastomoses encontrados em lesões malignas (Figura 8). A tipo II apresenta curva de realce formando um platô, que é uma combinação das curvas tipo I e III, e pode ser observada tanto em lesões benignas quanto malignas ${ }^{(\mathbf{7 , 2 0})}$ (Figura 9).
A caracterização de lesões mamárias pela curva tempo-intensidade apresenta sensibilidade de $91 \%$, especificidade de $83 \%$ e acurácia diagnóstica de $86 \%{ }^{(\mathbf{2 0})}$. Porém, deve-se ressaltar que a análise da cinética do realce não deve ser utilizada como critério diagnóstico isolado e sim de maneira

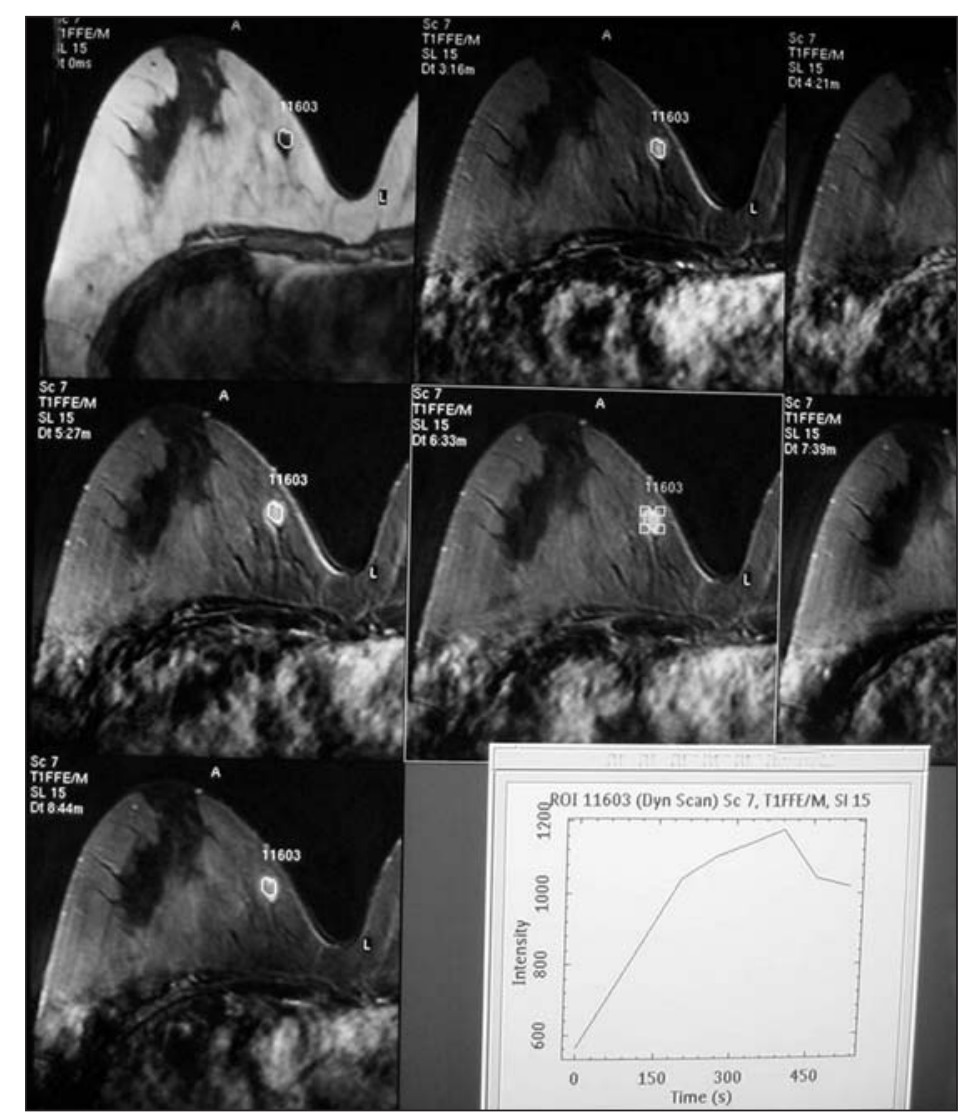

Figura 8. Seqüência gradiente-eco dinâmica ponderada em T1 obtida no plano axial antes, durante e após injeção endovenosa do contraste paramagnético, com medida de intensidade de sinal (ROI) sobre o nódulo e curva de realce tipo III.

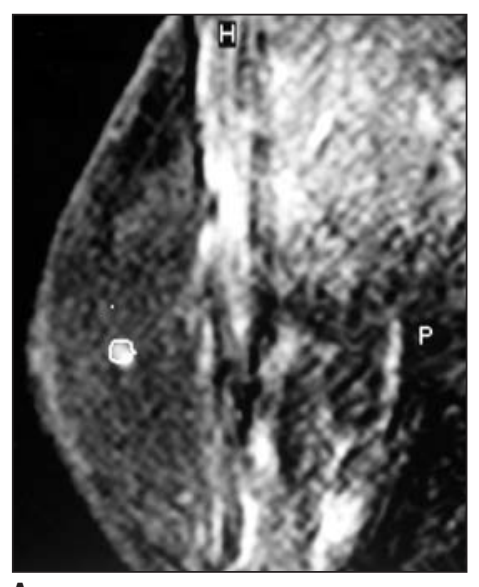

A

Figura 9. Nódulo sólido com curva de realce tipo II

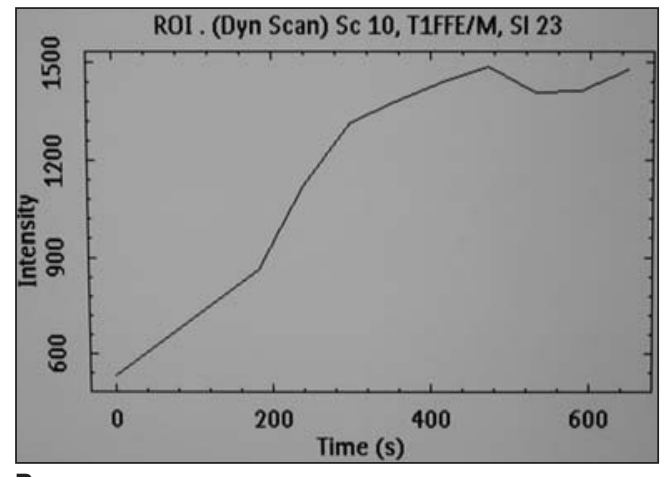

B 
integrada aos aspectos morfológicos no processo de diagnóstico diferencial das lesões mamárias (Quadro 1).

\section{ARTEFATOS}

Artefatos de movimentos respiratórios podem degradar a qualidade das imagens. O posicionamento da paciente em pronação minimiza os efeitos desses movimentos e reduz possível claustrofobia ${ }^{(\mathbf{1 , 3}, \mathbf{2 4})}$, que pode ocorrer em até $5 \%$ dos casos ${ }^{(36)}$.

Artefatos de batimentos cardíacos também degradam a qualidade das imagens e são visualizados como bandas que acompanham a direção da codificação de fase da imagem, principalmente quando se realizam seqüências no plano axial. Estes artefatos aumentam após a administração do contraste. Dependendo da direção do gradiente de codificação de fase, os artefatos podem se projetar sobre o tecido mamário que se localiza anteriormente ao coração ou sobre ambas as axilas (Figura 10). Dessa forma, dependendo da área de interesse, o eixo de codificação de fase deve ser alterado para deslocar-se o artefato para longe das estruturas de interesse $\mathrm{(24,25)}^{(25}$.

Os artefatos de movimentação corporal da paciente podem ser minimizados utilizando-se discreta compressão exercida sobre as mamas por alguns tipos de bobina durante o exame, promovendo, dessa maneira, a estabilização das mamas ${ }^{(\mathbf{1 , 2 4})}$. Essa compressão pode também ser gentilmente obtida utilizando-se suportes de tecido, espuma ou bolsas com conteúdo líquido.

Clipes metálicos na mama podem causar dificuldade de interpretação. Os clipes utilizados em cirurgias são geralmente de titânio e determinam artefatos de suscetibilidade magnética que se apresentam como área de ausência de sinal ("signal void") com alto sinal linear adjacente, que não deve ser interpretado como doença residual ou recorrente no sítio da quadrantectomia ${ }^{(7)}$. Existem também artefatos de suscetibilidade magnética relacionados a válvulas metálicas presentes em alguns expansores de tecido mamário (Figura 11).

\section{CONCLUSÃO}

A RM de mama com contraste teve progressos significativos desde sua introdução

Quadro 1 Diferenciação entre lesões benignas e malignas por meio da ressonância magnética de mama utilizando parâmetros morfológicos e comportamento de realce.

\begin{tabular}{|l|l|}
\hline Lesões benignas & \multicolumn{1}{c|}{ Lesões malignas } \\
\hline Contornos lisos & Contornos espiculados \\
Contornos lobulados & Contornos irregulares \\
Septações internas & Realce periférico \\
Ausência ou mínimo realce & Realce ductal \\
Curva de realce persistente ou progressivo & Curva de realce fugaz ou decrescente \\
\hline
\end{tabular}

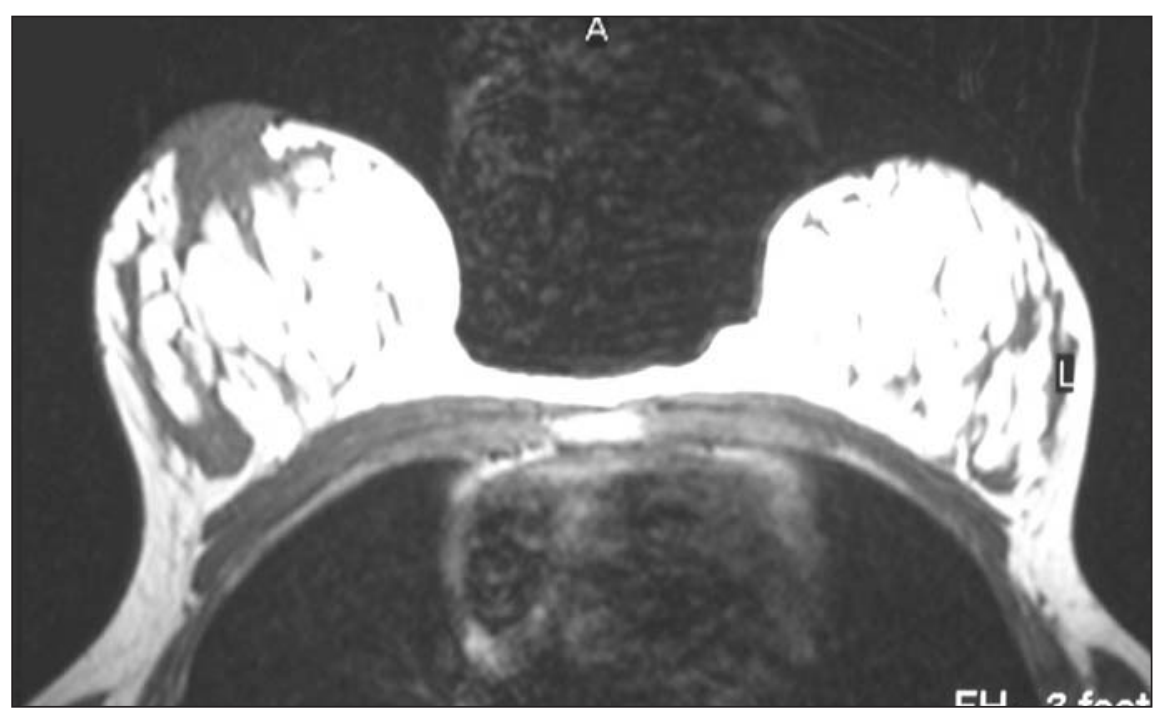

A

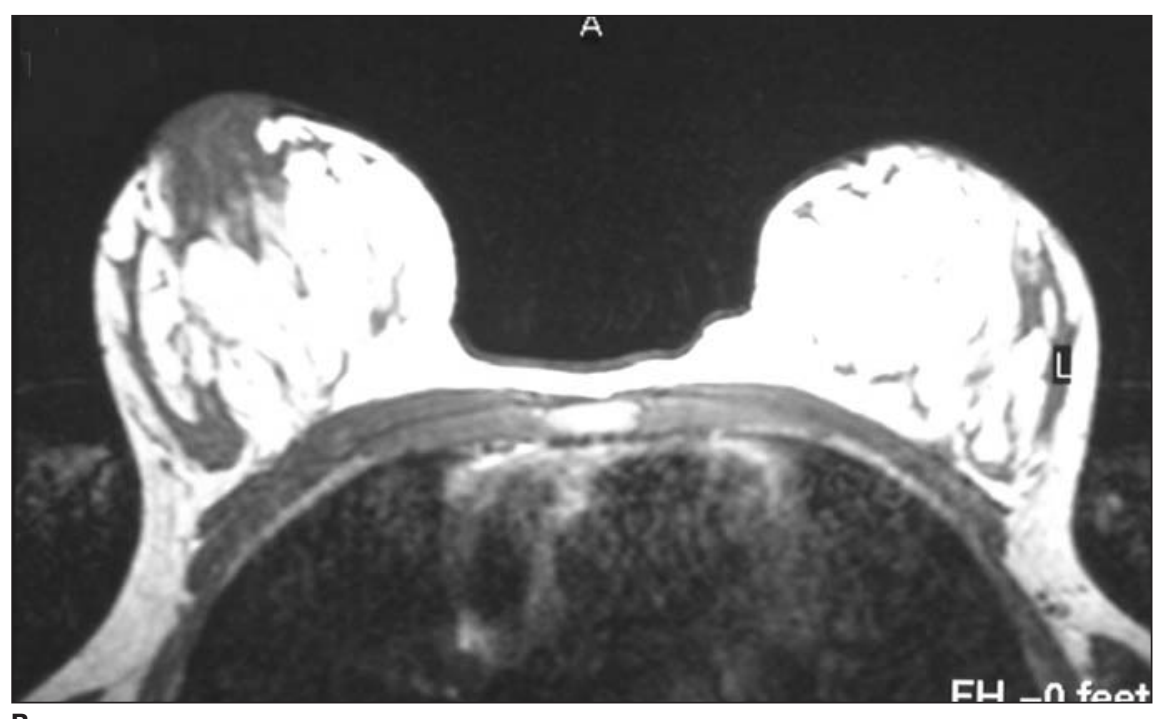

B

Figura 10. A: Imagens no plano axial ponderadas em T1 com técnica gradiente-eco. Nota-se artefato de batimento cardíaco projetando-se sobre a parede anterior do tórax. B: Artefato de batimento cardíaco projetando-se sobre as regiões axilares ao se mudar a direção de codificação de fase.

na prática radiológica. Porém, o uso da RM de mama na detecção, diagnóstico e estadiamento das mamas continua em desenvolvimento. E, como demonstrado através da revisão da literatura, ainda não temos até o momento uma técnica considerada padrão ou ideal para realização da RM de mama com contraste e critérios padronizados e universalmente aceitos para interpretação destes exames. A rápida e recente difusão do método certamente permitirá importantes avanços nesta direção. 


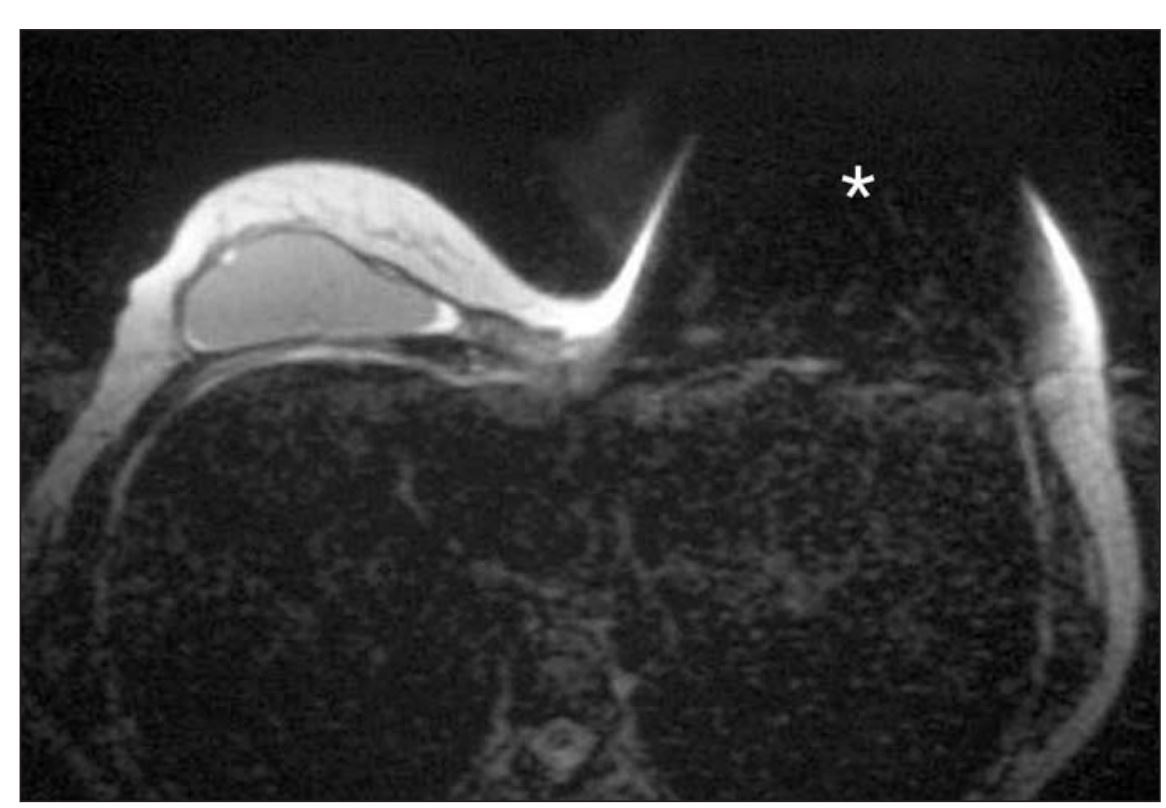

Figura 11. Imagem obtida no plano axial em paciente portadora de expansor mamário à esquerda, com válvula metálica produzindo grande quantidade de artefatos de suscetibilidade magnética distorcendo a imagem da mama esquerda (asterisco).

\section{REFERENCIAS}

1. Orel SG, Schnall MD. MR imaging of the breast for the detection, diagnosis, and staging of breast cancer. Radiology 2001;220:13-30.

2. Davis PL, McCarty KS Jr. Sensitivity of enhanced MRI for the detection of breast cancer: new, multicentric, residual, and recurrent. Eur Radiol 1997;7 Suppl 5:289-98.

3. Rankin SC. MRI of the breast. Br J Radiol 2000;73 806-18.

4. Schnall MD. Breast MR imaging. Radiol Clin North Am 2003;41:43-50.

5. Helbich TH. Contrast-enhanced magnetic resonance imaging of the breast. Eur J Radiol 2000;34:208 19.

6. Brown J, Buckley D, Coulthard A, et al. Magnetic resonance imaging screening in women at genetic risk of breast cancer: imaging and analysis protocol for the UK multicentre study. UK MRI Breast Screening Study Advisory Group. Magn Reson Imaging 2000;18:765-76.

7. Morris EA. Breast cancer imaging with MRI. Radiol Clin North Am 2002;40:443-66.

8. Kuhl CK, Kreft BP, Hauswirth A, et al. MR mammography at 0.5 tesla. I. Comparison of image quality and sensitivity of MR mammography at 0.5 and 1.5 T. Rofo 1995;162:381-9.

9. Heywang-Köbrunner SH, Bick U, Bradley WG Jr, et al. International investigation of breast MRI results of a multicentre study (11 sites) concerning diagnostic parameters for contrast-enhanced MRI based on 519 histopathologically correlated lesions. Eur Radiol 2001;11:531-46.

10. Sinha S, Sinha U, Lufkin RB. Instrumentos de res- sonância magnética. In: Lufkin RB, ed. Manual de ressonância magnética. Rio de Janeiro: Guanabara Koogan, 1999:90-105.

11. Pfleiderer SO, Reichenbach JR, Azhari T, Marx C, Wurdinger S, Kaiser WA. Dedicated double breast coil for magnetic resonance mammography imaging, biopsy, and preoperative localization. Invest Radiol 2003;38:1-8.

12. Warren R, Kessar P. A method of coil localization for breast lesions seen only on MRI. Br J Radiol 2001;74:548-51.

13. Konyer NB, Ramsay EA, Bronskill MJ, Plewes DB. Comparison of MR imaging breast coils. Radiology 2002;222:830-4.

14. Alvares B, Michell M. O uso da ressonância magnética na investigação do câncer mamário. Radiol Bras 2003;36:373-8.

15. Kirchin MA, Runge VM. Contrast agents for magnetic resonance imaging: safety update. Top Magn Reson Imaging 2003;14:426-35.

16. Liberman L, Morris EA, Dershaw DD, Abramson AF, Tan LK. Ductal enhancement on MR imaging of the breast. AJR 2003;181:519-25.

17. Tilanus-Linthorst MM, Obdeijn IM, Bartels KC, Koning HJ, Oudkerk M. First experiences in screening women at high risk for breast cancer with MR imaging. Breast Cancer Res Treat 2000;63:53-60.

18. Trecate G, Vergnaghi D, Bergonzi S, et al. Breast MRI screening in patients with increased familial and/or genetic risk for breast cancer: a preliminary experience. Tumori 2003;89:125-31.

19. Stoutjesdijk MJ, Boetes C, Jager GJ, et al. Magnetic resonance imaging and mammography in women with a hereditary risk of breast cancer. J Natl Cancer Inst 2001;93:1095-102.
20. Kuhl CK, Mielcareck P, Klaschik S, et al. Dynamic breast MR imaging: are signal intensity time course data useful for differential diagnosis of enhancing lesions? Radiology 1999; 211:101-10.

21. Heywang-Köbrunner SH, Haustein J, Pohl C, et al. Contrast-enhanced MR imaging of the breast: comparison of two different doses of gadopentetate dimeglumine. Radiology 1994;191:639-46.

22. Kinkel K, Helbich TH, Esserman LJ, et al. Dynamic high-spatial-resolution MR imaging of suspicious breast lesions: diagnostic criteria and interobserver variability. AJR 2000;175:35-43.

23. Nunes LW, Schnall MD, Orel SG. Update of breast MR imaging architectural interpretation model. Radiology 2001;219:484-94.

24. Hylton NM, Kinkel K. Technical aspects of breast magnetic resonance imaging. Top Magn Reson Imaging 1998;9:3-16.

25. Heywang-Köbrunner SH, Viehweg P. Breasts. In Stark DD, Bradley WG Jr, eds. Magnetic resonance imaging. 3rd ed. St. Louis: Mosby, 1999:307-19.

26. Knopp MV, Bourne MW, Sardanelli F, et al. Gadobenate dimeglumine-enhanced MRI of the breast: analysis of dose response and comparison with gadopentetate dimeglumine. AJR 2003; 181:66376.

27. Liu PF, Debatin JF, Caduff RF, Kacl G, Garzoli E, Krestin GP. Improved diagnostic accuracy in dynamic contrast enhanced MRI of the breast by combined quantitative and qualitative analysis. $\mathrm{Br} \mathrm{J}$ Radiol 1998;71:501-9.

28. Morris EA, Liberman L, Ballon DJ, et al. MRI of occult breast carcinoma in a high-risk population. AJR 2003;181:619-26.

29. Liberman L, Morris EA, Kim CM, et al. MR imaging findings in the contralateral breast of women with recently diagnosed breast cancer. AJR 2003; 180:333-41.

30. Kvistad KA, Rydland J, Vainio J, et al. Breast lesions: evaluation with dynamic contrast-enhanced T1-weighted MR imaging and with T2*-weighted first-pass perfusion MR imaging. Radiology 2000; 216:545-53.

31. Du W, Du YP, Bick U, et al. Breast MR imaging with high spectral and spatial resolutions: preliminary experience. Radiology 2002;224:577-85.

32. Delfaut EM, Beltran J, Johnson G, Rousseau J, Marchandise X, Cotten A. Fat suppression in MR imaging: techniques and pitfalls. RadioGraphics 1999; 19:373-82.

33. Jacobs MA, Barker PB, Bluemke DA, et al. Benign and malignant breast lesions: diagnosis with multiparametric MR imaging. Radiology 2003; 229:225-32.

34. Ikeda DM, Hylton NM, Kinkel K, et al. Development, standardization, and testing of a lexicon for reporting contrast-enhanced breast magnetic resonance imaging studies. J Magn Reson Imaging 2001;13:889-95.

35. Nunes LW, Schnall MD, Orel SG, et al. Breast MR imaging: interpretation model. Radiology 1997; 202:833-41.

36. Francis JM, Pennell DJ. Treatment of claustrophobia for cardiovascular magnetic resonance: use and effectiveness of mild sedation. J Cardiovasc Magn Reson 2000;2:139-41. 